\title{
EXISTENCE OF TRAVELING WAVE SOLUTIONS IN A HYPERBOLIC-ELLIPTIC SYSTEM OF EQUATIONS*
}

\author{
M. B. A. MANSOUR ${ }^{\dagger}$
}

Abstract. In this paper we discuss the existence of traveling wave solutions for a hyperbolicelliptic system of partial differential equations. The geometric theory of singular perturbations is employed.

Key words. Hyperbolic-elliptic system, Traveling wave solutions, Singular perturbations

Subject classifications. 35K65, 35B25, 34C37, 92C99.

\section{Introduction}

Over the last few decades a variety of mathematical models for studying aggregation cell movement has appeared. In fact, the active migration of cells is a significant feature of numerous biological phenomena ranging from wound healing, scar tissue formation, and tumor invasion to embryo implantation and organogenesis. Much of the mathematical modeling of cell migration has been predicated on the phenomena of reaction, diffusion, chemotaxis, haptotaxis, and mechanical interaction of cells, either singly or in combination (see $[4,5,8,11,12,13,16]$ ). Most of these mathematical models are in terms of parabolic (or coupling of parabolic and hyperbolic) partial differential equations or more general parabolic-elliptic system of partial differential equations.

In cell movement models, we consider the one-dimensional model [2], taking the form of a hyperbolic-elliptic system of partial differential equations within the region $\Omega(t)=\{x: 0 \leq \theta(t, x)<1\}$,

$$
\begin{gathered}
\partial_{t} \theta+\partial_{x}(\theta v)=f(\theta), \\
\partial_{x}\left(\mu(\theta) \partial_{x} v+s(\theta)\right)=\frac{\varphi \theta v}{1-\theta},
\end{gathered}
$$

with the nonlinearities

$$
\begin{aligned}
& f(\theta)=\eta \theta(1-\theta), \\
& \mu(\theta)=\mu_{0} \theta^{2}, \\
& s(\theta)=-\sigma \theta+\psi \theta^{2},
\end{aligned}
$$

where $\theta$ stands for the cell density, $v(t, x)$ stands for the cell velocity, $f(\theta), \mu(\theta), s(\theta)$ are the growth rate, the viscosity and the stress, respectively, and $\eta, \mu_{0}, \sigma, \psi, \varphi$ are positive constants.

With asymptotic boundary conditions as follows. The first boundary $(x \rightarrow \infty)$ :

$$
\theta=0, \text { and }|v| \text { is bounded }
$$

*Received: May 25, 2006; accepted (in revised version): September 8, 2006. Communicated by Shi Jin.

${ }^{\dagger}$ Department of Mathematics, Faculty of Science, South Valley University, Qena, Egypt (mah_mansour@hotmail.com). 
which imply zero flux boundary condition and the second boundary $(x \rightarrow-\infty)$ :

$$
\theta=1, v=0,
$$

again implying zero flux boundary condition. Notice that (1.1)-(1.2) with the above boundary conditions are the generalized compressible Stokes equations degenerating to the incompressible elliptic Stokes system in the region $\Re \backslash \Omega(t)=\{x: \theta(t, x) \equiv 1\}$. We refer the readers to [2] and [10] for a detailed derivation of the model and its biological background.

On the other hand, the natural biological object in cell movement models is the phenomenon of traveling waves. Based on the local stability analysis at the steady states of (1.1) and (1.2) and the numerical simulations, it was conjectured in [10] that (1.1) and (1.2) admit traveling wave solutions. Here we will use the geometric singular perturbation theory as well as the invariant manifold theory to give another proof for the existence of such solutions. We refer the readers to [10-16] for general literature of the geometric theory of singular perturbations and its applications.

The paper is organized as follows. In section 2, we describe how geometric singular perturbation theory is used to construct a locally invariant manifold $M_{\mu_{0}}$ for the traveling wave equations when $\mu_{0}$ is small. In section 3 we use this manifold to show the existence of a traveling wave solution. Section 4 contains conclusion.

\section{Geometric singular perturbation theory}

We are concerned with traveling wave solutions of system (1.1)-(1.2) with (1.3) connecting the steady states $(\theta=1, v=0)$ and $(\theta=0)$. Therefore the traveling wave solutions $(\theta(z), v(z))$ satisfy a system of ordinary differential equations in $z$

$$
\begin{gathered}
-c \frac{d \theta}{d z}+\frac{d}{d z}(\theta v)=f(\theta), \\
\frac{d}{d z}\left(\mu(\theta) \frac{d v}{d z}+s(\theta)\right)=\frac{\varphi \theta v}{1-\theta}, \theta<1,
\end{gathered}
$$

where $z=x-c t, c>0$ is a traveling wave speed. And boundary conditions

$$
\begin{aligned}
& \theta(z) \rightarrow 1, v(z) \rightarrow 0 \text { as } z \rightarrow-\infty, \\
& \theta(z) \rightarrow 0, v(z) \rightarrow v_{c} \text { as } z \rightarrow \infty,
\end{aligned}
$$

where $v_{c}$ is to be determined.

In order to apply the geometric theory of singular perturbations, we write (2.1)(2.2) into the following first order system:

$$
\begin{aligned}
\frac{d \theta}{d z} & =-\frac{f(\theta)+\theta q}{c-v}, \\
\frac{d v}{d z} & =-q, \\
\mu_{0} \theta \frac{d q}{d z} & =-\frac{\left(s^{\prime}(\theta)-2 \mu_{0} \theta q\right)(\eta(1-\theta)+q)}{c-v}-\frac{\varphi v}{1-\theta}, v \neq c,
\end{aligned}
$$

where' denotes the differentiation with respect to $\theta$. 
A traveling wave solution of the original system exists if there exists a heteroclinic orbit connecting the critical points of $(2.5)$.

Now, this system (2.5) has singularity at $\theta=1$. We can remove it by introducing the parameter $\tau$ such that $d \tau / d z=1 /(1-\theta(z))$. Except at $\theta=1$, where $d \tau / d z$ is not defined, $d \tau / d z>0$. Thus we have

$$
\theta(z)=\theta(\tau(z)), v(z)=v(\tau(z)), q(z)=q(\tau(z))
$$

Hence we have the system

$$
\begin{aligned}
\frac{d \theta}{d \tau} & =-\frac{(1-\theta)(f(\theta)+\theta q)}{c-v}, \\
\frac{d v}{d \tau} & =-(1-\theta) q, \\
\mu_{0} \theta \frac{d q}{d \tau} & =-\frac{(1-\theta)\left(s^{\prime}(\theta)-2 \mu_{0} \theta q\right)(\eta(1-\theta)+q)}{c-v}-\varphi v .
\end{aligned}
$$

Note that systems (2.5) and (2.7) are topologically equivalent in $\{(\theta, v, q) \mid \theta<1,-\infty<$ $v<\infty,-\infty<q<\infty\}$ and the critical points now are $\left(1,0, q_{0}\right)$ and $\left(0, v_{c}, 0\right)$ where $q_{0}$ is to be determined and $v_{c}=\frac{c}{2}-\sqrt{\left(\frac{c}{2}\right)^{2}-\frac{\eta \sigma}{\varphi}}$ is a solution of the equation $v^{2}-c v+\frac{\eta \sigma}{\varphi}=0$. This is true because (2.6) defines a re-parameterization of the orbits which preserves the orientation.

In this problem, $\mu_{0}>0$ is small, singular perturbation parameter and system (2.7) is a singularity perturbed system. One important component of the geometric theory of singular perturbations is the Fenichel's invariant manifold theorem which requires the normal hyperbolicity of the critical manifold in a singularity perturbed system [6]. Thus we introduce again the new parameter $\zeta: d \zeta / d \tau=1 / \mu_{0} \theta(\tau)$ to reduce $(2.7)$ to

$$
\begin{aligned}
& \frac{d \theta}{d \zeta}=-\frac{\mu_{0} \theta(1-\theta)(f(\theta)+\theta q)}{c-v}, \\
& \frac{d v}{d \zeta}=-\mu_{0} \theta(1-\theta) q, \\
& \frac{d q}{d \zeta}=-\frac{(1-\theta)\left(s^{\prime}(\theta)-2 \mu_{0} \theta q\right)(\eta(1-\theta)+q)}{c-v}-\varphi v .
\end{aligned}
$$

While these two systems are equivalent for $\mu_{0}>0$, the different time-scales give rise to different limiting systems. Letting $\mu_{0} \rightarrow 0$ in (2.7), we obtain

$$
\begin{aligned}
\frac{d \theta}{d \tau} & =-\frac{(1-\theta)(f(\theta)+\theta q)}{c-v}, \\
\frac{d v}{d \tau} & =-(1-\theta) q \\
0 & =-\frac{(1-\theta)\left(s \prime(\theta)-2 \mu_{0} \theta q\right)(\eta(1-\theta)+q)}{c-v}-\varphi v .
\end{aligned}
$$

Thus the flow of system (2.9) is confined to the set

$$
M_{0}:=\left\{(\theta, v, q) \in \mathbb{R}^{3}: q=-\frac{\varphi v(c-v)}{(1-\theta) s^{\prime}(\theta)}-\eta(1-\theta)\right\}
$$

and its dynamics are determined by the first two equations only. 
On the other hand, setting $\mu_{0} \rightarrow 0$ in (2.8) results in the system

$$
\begin{aligned}
& \frac{d \theta}{d \zeta}=0, \\
& \frac{d v}{d \zeta}=0, \\
& \frac{d q}{d \zeta}=-\frac{(1-\theta)\left(s^{\prime}(\theta)-2 \mu_{0} \theta q\right)(\eta(1-\theta)+q)}{c-v}-\varphi v .
\end{aligned}
$$

Any points in $M_{0}$ are the equilibria of system (2.11). Generally, system (2.7) is referred to as the slow system, since the time-scale $\tau$ is slow, and (2.8) is referred to as the fast system, since the time-scale $\zeta$ is fast. Hence $\theta$ and $v$ are called slow variable and $q$ is called the fast variable. $M_{0}$ is the slow manifold.

If $M_{0}$ is normally hyperbolic, then we can use the geometric singular perturbation theory of Fenichel to obtain a two dimensional invariant manifold $M_{\mu_{0}}$ for the flow when $\mu_{0}>0$, which implies the persistence of the slow manifold as well as the stable and unstable foliations. As a consequence the dynamics in the vicinity of the slow manifold are completely determined by the one on the slow manifold. Therefore, we only need to study the flow of the slow manifold (2.7) restricted to $M_{\mu_{0}}$ and show that the two-dimensional reduced system has a heteroclinic orbit.

Recall that $M_{0}$ is normally hyperbolic manifold if the linearization of the fast system (2.8), restricted to $M_{0}$, has exactly $\operatorname{dim} M_{0}$ eigenvalues with zero real part. matrix

The linearization of the fast system (2.8), restricted to $M_{0}$ (i.e. $\mu_{0}=0$ ) has the

$$
\left(\begin{array}{ccc}
0 & 0 & 0 \\
0 & 0 & 0 \\
I_{\theta} & I_{v} & I_{q}
\end{array}\right)
$$

at the point $(\theta, v, q) \in M_{0}$, where

$$
\begin{aligned}
& I_{\theta}=\frac{2 \eta(1-\theta) s^{\prime}(\theta)+s^{\prime}(\theta) q-2 \psi(1-\theta)(\eta(1-\theta)+q)}{c-v}, \\
& I_{v}=-\frac{(1-\theta) s^{\prime}(\theta)(\eta(1-\theta)+q)}{(c-v)^{2}}-\varphi, \\
& I_{q}=-\frac{(1-\theta) s^{\prime}(\theta)}{c-v} .
\end{aligned}
$$

Its set of eigenvalues is $\left\{0,0, \lambda_{3}\right\}$ where $\lambda_{3}=-\frac{(1-\theta)(-\sigma+2 \psi \theta)}{c-v}, \theta<1, v \neq c$. Only the two zero eigenvalues have eigenvectors tangent to $M_{0}$. Thus $M_{0}$ satisfies the normal hyperbolic condition required by the Fenichel's theorem, and hence there exists an invariant manifold $M_{\mu_{0}}$. To determine $M_{\mu_{0}}$, explicitly, we have

$$
M_{\mu_{0}}:=\left\{(\theta, v, q) \in \mathbb{R}^{3}: q=g\left(\theta, v, \mu_{0}\right)\right\},
$$

where the function $g$ depends smoothly on $\mu_{0}$, is to be determined and satisfies

$$
g(\theta, v, 0)=-\frac{\varphi v(c-v)}{(1-\theta) s^{\prime}(\theta)}-\eta(1-\theta) .
$$


Substitution of $g$ into (2.7), to obtain a differential equation system on $M_{\mu_{0}}$, results in a regular perturbation. In other words, on $M_{\mu_{0}},(2.7)$ reduces to

$$
\begin{aligned}
& \frac{d \theta}{d \tau}=-\frac{(1-\theta)\left(f(\theta)+\theta g\left(\theta, v, \mu_{0}\right)\right)}{c-v}, \\
& \frac{d v}{d \tau}=-(1-\theta) g\left(\theta, v, \mu_{0}\right),
\end{aligned}
$$

where

$$
g\left(\theta, v, \mu_{0}\right)=g(\theta, v, 0)+\mu_{0} g_{1}(\theta, v, 0)+\mathrm{O}\left(\mu_{0}^{2}\right),
$$

and

$$
g_{1}(\theta, v, 0)=\frac{2 \varphi \theta v}{(1-\theta) s^{\prime}(\theta)}\left(\frac{\varphi v(c-v)}{s^{\prime}(\theta)}+\eta(1-\theta)^{2}\right) .
$$

(2.14) can be written as

$$
\begin{aligned}
& \frac{d \theta}{d \tau}=\frac{\varphi \theta v}{s^{\prime}(\theta)}-\mu_{0} \frac{\theta(1-\theta)}{c-v} g_{1}(\theta, v, 0)+\mathrm{O}\left(\mu_{0}^{2}\right), \\
& \frac{d v}{d \tau}=\frac{\varphi v(c-v)}{s^{\prime}(\theta)}+\eta(1-\theta)^{2}-\mu_{0}(1-\theta) g_{1}(\theta, v, 0)+\mathrm{O}\left(\mu_{0}^{2}\right),
\end{aligned}
$$

which determines the dynamics on the slow manifold $M_{\mu_{0}}$. We shall study this system in the next section.

\section{The flow on the manifold $M_{\mu_{0}}$}

When $\mu_{0}=0$, system (2.15) reduces to the corresponding system of ODEs

$$
\begin{aligned}
& \frac{d \theta}{d \tau}=\frac{\varphi \theta v}{s^{\prime}(\theta)}, \\
& \frac{d v}{d \tau}=\frac{\varphi v(c-v)}{s^{\prime}(\theta)}+\eta(1-\theta)^{2},
\end{aligned}
$$

which are phase plane equations in traveling wave problem. This system (3.1) has two equilibria, $P_{1}=(1,0)$ and $P_{0}=\left(0, v_{c}\right)$ where $v_{c}=\frac{c}{2}-\sqrt{\left(\frac{c}{2}\right)^{2}-\frac{\eta \sigma}{\varphi}}$. We note that there is another solution $v_{c}=\frac{c}{2}+\sqrt{\left(\frac{c}{2}\right)^{2}-\frac{\eta \sigma}{\varphi}}$ but we excluded, as before. The following result shows that there is a traveling wave solution of (3.1) connecting $P_{0}$ and $P_{1}$.

THEOREM 3.1. If $c \geq 2 \sqrt{\frac{\eta \sigma}{\varphi}}$, then in the $(\theta, v)$ phase plane for system (3.1), there is a heteroclinic orbit connecting the critical points $P_{0}$ and $P_{1}$ and hence traveling wave solution $(\theta, v)(\tau)$ connecting $\left(0, v_{c}\right)$ and $(1,0)$ with $\theta(\tau)$ is strictly monotonically decreasing.

Proof. Linear analysis of system (3.1) about $P_{0}$ shows that the determinant of the Jacobian matrix is $\frac{\varphi^{2} v_{c}}{\sigma^{2}}\left(c-2 v_{c}\right)>0$ (under the condition on $c$ ). Hence $P_{0}$ is a node. The trace of the Jacobian is $\frac{\varphi}{\sigma}\left(c-v_{c}\right)<0$, so $P_{0}$ is a stable node. The eigenvalues of the Jacobian are $\lambda_{1}=-\frac{\varphi v_{c}}{\sigma}, \lambda_{2}=-\frac{\varphi}{\sigma}\left(c-2 v_{c}\right)$. Both are negative. Linear analysis of (3.1) about $P_{1}$ shows that the determinant of the Jacobian is zero. The trace of the Jacobian is $-\frac{\varphi c}{-\sigma+2 \psi}<0, \sigma<\psi$, hence $P_{1}$ is a non-hyperbolic point and the eigenvalues 
of the Jacobian matrix associated with the system (3.1) at $P_{1}$ are $\lambda_{1}=0, \lambda_{2}=\frac{\varphi c}{-\sigma+2 \psi}$. The corresponding eigenvectors are $v_{1}=(1,0)$ and $v_{2}=(1, c)$, respectively. From the Centre Manifold Theorem we have that (3.1) has a one-dimensional unstable manifold locally tangent to the eigenvector $v_{2}$ and a one-dimensional center manifold locally tangent to the eigenvector $v_{1}$. More precisely, the approximate equation of the center manifold is given by

$$
v=-\frac{\eta(-\sigma+2 \psi)}{\varphi c}(1-\theta)^{2}+O\left((1-\theta)^{3}\right)
$$

and the equation of the unstable manifold locally around $P_{1}$ is

$$
v=-c(1-\theta)-\left(c-\frac{\eta(-\sigma+2 \psi)}{2 \varphi c}\right)(1-\theta)^{2}+O\left((1-\theta)^{3}\right)
$$

implying that the system (3.1) is weakly repelling on both of these manifolds. Therefore, we conclude that $P_{1}$ is a weak (i.e., non-hyperbolic) node. Note that this also comes more clearly if we put $\vartheta=1-\theta$ and work in the $(\vartheta, v)$ phase plane. Thus, any traveling wave solution originates from $P_{1}$, whose path is tangent to the center manifold.

All that remains now is to establish the existence of a heteroclinic orbit connecting the two equilibria $P_{1}$ and $P_{0}$. To do this, we use the continuity property of solutions of (3.1) with respect to the parameter $c$ as well as the monotonicity property of the paths of the trajectories of (3.1). Then, we shall use the vector field defined by (3.1), the vertical nullclines of (3.1) and the local analysis of (3.1) at $P_{0}$ and $P_{1}$.

From (3.1), we have $v$-nullclines, $\dot{v}=0$ only on the graph of the following two functions:

$$
\begin{aligned}
& V_{1}(\theta)=\frac{c}{2}-\sqrt{\left(\frac{c}{2}\right)^{2}+\frac{\eta}{\varphi}(1-\theta)^{2} s^{\prime}(\theta)}, \\
& V_{2}(\theta)=\frac{c}{2}+\sqrt{\left(\frac{c}{2}\right)^{2}+\frac{\eta}{\varphi}(1-\theta)^{2} s^{\prime}(\theta)} .
\end{aligned}
$$

We are interested only in $V_{1}(\theta)$. Thus, let us list some properties of $V_{1}$

$$
\begin{aligned}
& 1-V_{1}(0)=\frac{c}{2}-\sqrt{\left(\frac{c}{2}\right)^{2}-\frac{\eta \sigma}{\varphi}}=v_{c}, \text { and } V_{1}(1)=0, \\
& 2-\frac{d V_{1}}{d \theta}(0)=-\frac{\eta(\sigma+\psi)}{\varphi \sqrt{\left(\frac{c}{2}\right)^{2}-\frac{\eta \sigma}{\varphi}}}<0, \text { and } \frac{d V_{1}}{d \theta}(1)=0
\end{aligned}
$$

We note that for given parameters, it is clear that the shape of $V_{1}(\theta)$ changes with $c$. Further, let $W^{u}\left(P_{1}\right)$ be the unstable manifold at $P_{1}$. Then from a continuity argument, it follows that the unstable manifold $W^{u}\left(P_{1}\right)$ leaves $P_{1}$ and enters the region

$$
\Re=\left\{(\theta, v): 0<\theta<1, V_{1}(\theta) \leq v<v_{c}\right\},
$$

where the vector field defined by the right-hand sides of system (3.1) pushes it towards the graph of $V_{1}$. Therefore, the vector field pushes $W^{u}\left(P_{1}\right)$ towards $P_{0}$ and hence $W^{u}\left(P_{1}\right)$ reaches $P_{0}$ as $\tau$ tends to $+\infty$, and is the unique orbit connecting the point $P_{1}$ to the point $P_{0}$. It follows also that $W^{u}\left(P_{1}\right)$ ends at $P_{0}$ by defining an invariant set of (3.1) and by using the Paincare-Bendixon theorem. Hence there exists a heteroclinc 
orbit connecting $P_{1}$ with $P_{0}$, and traveling wave solution that approaches both end limits exponentially fast, thus concluding the the proof.

Now we can see that for $\mu_{0}>0$, system (2.15) still has same critical points $P_{0}$ and $P_{1}$. These two points correspond to the two critical points $\left(0, v_{c}, 0\right)$ and $\left(1,0, q_{0}\right)$ of the full system (2.7). The following theorem shows that there is a heteroclinic orbit connecting $P_{0}$ and $P_{1}$ and thus equation (2.15) has a traveling wave solution connecting $\left(0, v_{c}\right)$ and $(1,0)$.

THEOREM 3.2. For any $\mu_{0}>0$ sufficiently small, there exists a speed $c$ such that the system (2.15) has a heteroclinic orbit connecting the two critical points $\left(0, v_{c}\right)$ and $(1,0)$.

Proof. We write system (2.15) as

$$
\begin{aligned}
& \frac{d \theta}{d z}=\Phi\left(\theta, v, c, \mu_{0}\right), \\
& \frac{d v}{d z}=\Psi\left(\theta, v, c, \mu_{0}\right) .
\end{aligned}
$$

Note that

$$
\Phi(\theta, v, c, 0)=\frac{\varphi \theta v}{s^{\prime}(\theta)}
$$

and

$$
\Psi(\theta, v, c, 0)=\frac{\varphi v(c-v)}{s^{\prime}(\theta)}+\eta(1-\theta)^{2}
$$

We know that when $\mu_{0}=0$ the traveling wave solution $(\theta, v)(\tau)$ connecting $\left(0, v_{c}\right)$ and $(1,0)$, with $\theta(\tau)$ is strictly monotone, exists if its speed $c \geq 2 \sqrt{\frac{\eta \sigma}{\varphi}}$. Therefore, in the $(\theta, v)$ plane, it can be characterized as the graph of some function,i.e.

$$
V=w(\theta, c)
$$

By the stable manifold theorem, for sufficiently small $\mu_{0}$, we can also characterize the unstable manifold at $(1,0)$ as the graph of some function,

$$
V=w_{1}\left(\theta, c, \mu_{0}\right),
$$

where $w_{1}\left(1, c, \mu_{0}\right)=0$. Furthermore, by continuous dependence of the solutions on parameters, This manifold must cross the line $\theta=1 / 2$ somewhere if $\mu_{0}$ is sufficiently small.

Similarly, let $V=w_{2}\left(\theta, c, \mu_{0}\right)$ be the equation for the stable manifold at $\left(0, v_{c}\right)$. Clearly, $w_{2}\left(0, c, \mu_{0}\right)=v_{c}$, and it also crosses the line $\theta=1 / 2$ somewhere for suitably small $\mu_{0}$. Thus

$$
w_{1}(\theta, c, 0)=w_{2}(\theta, c, 0)=w(\theta, c) .
$$

For $\mu_{0}=0$, fix $c=c_{0} \geq 2 \sqrt{\frac{\eta \sigma}{\varphi}}$, so that the equation of the corresponding wave in the phase plane is $V=\left(\theta, C_{0}\right)$. To show that there is a heteroclinic connection when $\mu_{0}>0$, we use a similar argument used in [1] and [14], that is, to show that there exists a 
unique value of $c=c\left(\mu_{0}\right)$, near $c_{0}$, such that the manifolds $w_{1}$ and $w_{2}$ cross the line $\theta=1 / 2$ at the same point. Define

$$
h\left(c, \mu_{0}\right)=w_{2}\left(\frac{1}{2}, c, \mu_{0}\right)-w_{1}\left(\frac{1}{2}, c, \mu_{0}\right) .
$$

Note that both $V=w_{1}\left(\theta, c, \mu_{0}\right)$ and $V=w_{2}\left(\theta, c, \mu_{0}\right)$ satisfy the equation

$$
\frac{d V}{d \theta}=\frac{\Psi\left(\theta, v, c, \mu_{0}\right)}{\Phi\left(\theta, v, c, \mu_{0}\right)}
$$

We have

$$
\begin{aligned}
\frac{d}{d \theta}\left(\frac{\partial w_{1}}{\partial c}(\theta, c, 0)\right) & =\left.\frac{\partial}{\partial c}\left(\frac{d w_{1}}{d \theta}(\theta, c, 0)\right)\right|_{c=c_{0}} \\
& =\left.\frac{\partial}{\partial c}\left(\frac{\Psi\left(\theta, w_{1}(\theta, c, 0), c, 0\right)}{\Phi\left(\theta, w_{1}(\theta, c, 0), c, 0\right)}\right)\right|_{c=c_{0}} \\
& =\left.\frac{\partial}{\partial c}\left(\frac{\varphi c w_{1}(\theta, c, 0)-\varphi w_{1}^{2}(\theta, c, 0)+\eta(1-\theta)^{2} s^{\prime}(\theta)}{\varphi \theta w_{1}(\theta, c, 0)}\right)\right|_{c=c_{0}} \\
& =\left.\frac{\partial}{\partial c}\left(\frac{c}{\theta}-\frac{w_{1}(\theta, c, 0)}{\theta}+\frac{\eta(1-\theta)^{2} s^{\prime}(\theta)}{\varphi \theta w_{1}(\theta, c, 0)}\right)\right|_{c=c_{0}} \\
& =\frac{1}{\theta}-\left(\frac{1}{\theta}+\frac{\eta(1-\theta)^{2} s^{\prime}(\theta)}{\varphi \theta w^{2}\left(\theta, c_{0}, 0\right)}\right) \frac{\partial w_{1}}{\partial c}\left(\theta, c_{0}, 0\right) .
\end{aligned}
$$

Integrating from $\theta=1 / 2$ to $\theta=1$, we have

$$
\frac{\partial w_{1}}{\partial c}\left(\frac{1}{2}, c_{0}, 0\right)=-2 \int_{1 / 2}^{1} \exp \left[\int_{1 / 2}^{\alpha} \frac{\eta(1-\rho)^{2} s^{\prime}(\rho)}{\varphi \rho w^{2}\left(\rho, c_{0}, 0\right)} d \rho\right] d \alpha .
$$

Similarly, we have

$$
\frac{d}{d \theta}\left(\frac{\partial w_{2}}{\partial c}(\theta, c, 0)\right)=\frac{1}{\theta}-\left(\frac{1}{\theta}+\frac{\eta(1-\theta)^{2} s^{\prime}(\theta)}{\varphi \theta w^{2}\left(\theta, c_{0}, 0\right)}\right) \frac{\partial w_{2}}{\partial c}\left(\theta, c_{0}, 0\right) .
$$

Integrating from $\theta=0$ to $\theta=1 / 2$ yields

$$
\frac{\partial w_{2}}{\partial c}\left(\frac{1}{2}, c_{0}, 0\right)=2 \lim _{\delta \rightarrow 0} \int_{\delta}^{1 / 2} \exp \left[\int_{1 / 2}^{\alpha} \frac{\eta(1-\rho)^{2} s^{\prime}(\rho)}{\varphi \rho w^{2}\left(\rho, c_{0}, 0\right)} d \rho\right] d \alpha .
$$

Combining (3.5) and (3.6), we have

$$
\begin{aligned}
\frac{\partial h}{\partial c}\left(c_{0}, 0\right) & =\frac{\partial w_{2}}{\partial c}\left(\frac{1}{2}, c_{0}, 0\right)-\frac{\partial w_{1}}{\partial c}\left(\frac{1}{2}, c_{0}, 0\right) \\
& =2 \lim _{\delta \rightarrow 0} \int_{\delta}^{1} \exp \left[\int_{1 / 2}^{\alpha} \frac{\eta(1-\rho)^{2} s^{\prime}(\rho)}{\varphi \rho w^{2}\left(\rho, c_{0}, 0\right)} d \rho\right] d \alpha>0 .
\end{aligned}
$$

Thus, by implicit function theorem, for sufficiently small $\mu_{0}, h\left(c, \mu_{0}\right)=0$ has root $c=c\left(\mu_{0}\right)$ near $c_{0}$. This implies that the manifolds $w_{1}$ and $w_{2}$ cross the line $\theta=1 / 2$ at the same point. This establishes the existence of a heteroclinic connection. 


\section{Conclusion}

In this paper we have considered a simple mathematical model of cell migration. The model takes the form of hyperbolic-elliptic system of partial differential equations and has many applications such as wound healing and tumor growth. With the methods of geometric singular perturbation theory as developed in [7] and [6] and used in [1], [3], and [14], we have studied the existence of traveling wave solutions for the model system. We have constructed a locally invariant manifold $M_{\mu_{0}}$ for traveling wave equations when $\mu_{0}$ is small and positive. Then we used this manifold to show the existence of a traveling wave solution.

Acknowledgement. The author thanks the anonymous referee for helpful comments and suggestions.

\section{REFERENCES}

[1] M. E. Akveld and J. Hulshof, Travelling wave solutions of a fourth-order semilinear diffusion equation, Appl. Math. Letters, 11, 115-120, 1998

[2] W. Alt and M. Dembo, Cytoplasm dynamics and cell motion: two-phase flow models, Math. Biosci., 156, 207-228, 1999.

[3] P. Ashwin, M. V. Bbartuccelli, T. J. Bridges and S. A. Gourley, Travelling fronts fot the KPPFisher equation with spatio-temporal delay, Z. Angew. Math. Phys., 53, 103-122, 2002

[4] H. M. Byrne, M. A. J. Chaplain, G. J. Pettet and D. L. S. McElwain, A mathematical model of trophoblast invasion, J. Theoret. Med., 1, 275-286, 1999.

[5] G. C. Cruywagen and J. D. Murray, On a tissue interaction model for skin pattern formation, J. Nonlin. Sci., 2, 217-240, 1992.

[6] N. Fenichel, Geometric singular perturbation theory for ordinary differential equations, J. Diff. Eqn., 31, 53-98, 1979.

[7] C. K. R. T. Jones, Geometric Singular Perturbation Theory, Dynamical Systems, eds. R. Johnson, Springer-Verlag, Berlin Heidelberg, 1995.

[8] J. A. Landman, G. J. Pettet and D. F. Newgrene, Chemotactic cellular migration: smooth and discontinuous travelling wave solutions, SIAM J. Appl. Math., 63, 1666-1681, 2003.

[9] X.-B. Lin, Asymptotic expansion for layer solutions of a singularity perturbed reaction-diffusion system, Trans. Amer. Math. Soc., 348, 713-753, 1996.

[10] M. B. A. Mansour, Singularity analysis for travelling wave solutions of degenerate diffusion and transport equations modelling cell motility, PhD Thesis, University of Bonn, 2001.

[11] J. D. Murray, Mathematical Biology, 2nd ed., Springer-Verlag, Heidelberg, 1993.

[12] M. E. Orme and M. A. J. Chaplain, A mathematical model of vascular tumour growth and invasion, Math. Comput. Modell. 23, 43-60, 1996.

[13] G. J. Pettet, H. M. Byrne, D. L. S. McElwain and J. Norbury, A model of wound-healing angiogenesis in soft tissue, Math. Biosci., 136, 35-63, 1996.

[14] S. Ruan and D. Xiao, Stability of steady states and existence of travelling waves in a vectordisease model, Proc. R. Soc. Edinb. A 134, 991-1011, 2004.

[15] K. Sakamoto, Invariant manifold in singular perturbation problems for ordinary differential equations, Proc. R. Soc. Edinb. A, 116, 45-78, 1990.

[16] J. A. Sherratt and J. D. Murray, Mathematical analysis of a basic model for epidermal wound healing, J. Math. Biol., 29, 389-404, 1991. 